\title{
Thrombotic thrombocytopenic purpura and systemic lupus erythematosus
}

\author{
DAVID A FOX, JAMES D FAIX, JON COBLYN, PATRICIA FRASER, \\ BRIAN SMITH, AND MICHAEL E WEINBLATT
}

From the Departments of Rheumatology and Immunology, Pathology, and Medicine, Brigham and Women's and Beth Israel Hospitals, Harvard Medical School, Boston, Massachusetts

SUMmARY We report two patients with systemic lupus erythematosus who subsequently developed thrombotic thrombocytopenic purpura. In each case the coexistence of these two conditions was confirmed by pathological findings. Both patients responded to treatment, but one eventually died. A review of the literature suggests a possible relationship between the two disorders.

Key words: rheumatology, blood coagulation disorders, collagen diseases, plasmapheresis, cyclophosphamide.

Thrombotic thrombocytopenic purpura (TTP) is a rare clinical syndrome of unknown cause characterised by the pentad of microangiopathic haemolytic anaemia, thrombocytopenic purpura, neurological abnormalities, fever, and renal dysfunction. ${ }^{1-3}$ Patients with systemic lupus erythematosus (SLE), however, may develop similar clinical findings. We report two patients with pre-existing SLE whose course was complicated by the development of TTP.

\section{Case reports}

CASE 1

A 40 year old woman was diagnosed in 1977 as having seropositive rheumatoid arthritis and was treated with anti-inflammatory agents and sodium aurothiomalate. Over the next 18 months she developed a malar rash, Raynaud's phenomenon, alopecia, and a positive antinuclear antibody (ANA) test. In September 1979 she was hospitalised with fever, headache, purpura, a malar rash, petechiae, hepatosplenomegaly, and symmetrical polyarthritis. The urinalysis showed $3+$ proteinuria and haematuria without red cell casts. Haemoglobin was $68 \mathrm{~g} / \mathrm{l}$, white blood cell count $8000 / \mathrm{mm}^{3}$ $\left(8 \times 10^{9} / 1\right)$ with a left shift, platelet count $13000 / \mathrm{mm}^{3}$

Accepted for publication 27 September 1985.

Correspondence to Dr David A Fox. University of Michigan Medical Center, Department of Internal Medicine, Division of Rheumatology, Rackham Arthritis Research Unit, Ann Arbor, Michigan 48109. USA. $\left(13 \times 10^{9} / 1\right)$, reticulocyte count $14 \%$, and sedimentation rate $130 \mathrm{~mm} / 1 \mathrm{st} \mathrm{h}$. The peripheral smear showed many schistocytes and nucleated red cells. Prothrombin time and partial thromboplastin time were normal. Biochemical tests included creatinine $0.8 \mathrm{mg} / \mathrm{dl}(71 \mu \mathrm{mol} / \mathrm{l})$, lactic dehydrogenase (LD) $1120 \mathrm{IU} / \mathrm{l}$, and bilirubin $6.4 \mathrm{mg} / \mathrm{dl}(109 \mu \mathrm{mol} / \mathrm{l})$. The ANA test was positive at a titre of $1 / 40$ with a peripheral and diffuse pattern. Anti-double stranded DNA antibodies were not detected. Total haemolytic complement, $\mathrm{C} 3$, and $\mathrm{C} 4$ were all depressed. Direct and indirect Coombs' tests and assays for antiplatelet antibodies were negative. A diagnosis of thrombotic thrombocytopenic purpura was made, though a gingival biopsy was negative. Treatment was initiated with methylprednisolone $100 \mathrm{mg} /$ day, aspirin, dipyridamole, and multiple courses of plasma exchange. Despite these measures there was no improvement in the patient's clinical or haematological parameters, and a splenectomy was performed. Temporary improvement followed, with stabilisation of her haemoglobin and platelet count. Three days after discharge the patient relapsed with fever, haemolytic anaemia, and profound thrombocytopenia. She improved with plasmapheresis and methylprednisolone ( $100 \mathrm{mg} /$ day $)$, and over the next few months the amount of steroids was slowly reduced and eventually discontinued. Two months later she again relapsed. She responded to a repeat course of high dose steroids combined with plasma infusions but developed complications of steroid 
therapy, including hypertension and hyperglycaemia. Azathioprine was added when a reduction in steroid dose was followed by another relapse. Despite temporary improvement thrombocytopenia and fever recurred, and cyclophosphamide was begun at a dose of $200 \mathrm{mg}$ daily. A clinical and haematological remission of the patient's TTP has since been maintained for over five years. For 42 months she received prednisone, $5 \mathrm{mg}$ every other day, and daily cyclophosphamide in slowly tapering doses. Both medications were eventually discontinued without further flares of TTP. Her polyarthritis has subsided and there is no evidence of active SLE.

CASE 2

A 42 year old women developed SLE in 1977, manifested by polyarthritis, Raynaud's phenomenon, malar rash, pleuritis, membranous glomerulonephritis, and an ANA titre of 1/10 000 with a homogeneous and speckled pattern. Antibody to double stranded DNA was present and complement components were depressed. She was treated with prednisone and improved, but over the next three years she was hospitalised on multiple occasions for fever, psychosis, and seizures. In November 1981 she was hospitalised because of three days of headache and abdominal pain, preceded by one week of arthralgias, myalgias, and malaise. Her temperature was $103^{\circ} \mathrm{F}$, and palatal and conjunctival haemorrhages were present. Alopecia and superficial pharyngeal erosions were noted. The haemoglobin was $69 \mathrm{~g} / \mathrm{l}$, platelet count $9000 / \mathrm{mm}^{3}\left(9 \times 19^{9} / 1\right)$, and white blood cell count $17000 / \mathrm{mm}^{3}\left(17 \times 19^{9} / 1\right)$. Total haemolytic complement, C3, and C4 were normal, though hypocomplementaemia had been present during previous SLE flares. Prothrombin time and partial thromboplastin time were normal. The peripheral blood smear showed many schistocytes and the LD was markedly raised. Bone marrow examination showed hyperplasia of all cell lines. The diagnosis of TTP was confirmed by gingival biopsy.

Treatment was initiated with methylprednisolone $60 \mathrm{mg} /$ day, aspirin $1200 \mathrm{mg} /$ day, dipyridamole 600 $\mathrm{mg} / \mathrm{day}$, and multiple courses of plasmapheresis. Over the next week the platelet count normalised and the peripheral smear, haemoglobin, and reticulocyte count improved. A sudden episode of aphasia occurred but resolved after plasmapheresis. The patient remained stable after discharge from the hospital on prednisone, aspirin, and dipyridamole. In late December 1981 she abruptly stopped all medication and one week later developed fever, acute abdominal pain, profound thrombocytopenia, and anaemia. The peripheral smear again showed many schistocytes and helmet cells. Despite corti- $\frac{\square}{\varphi}$. costeroids, antiplatelet agents, and plasmapheresis the patient died.

\section{Pathology}

Characteristic histopathological features of both $\stackrel{\frac{\sigma}{\partial}}{\circ}$ TTP and SLE were present in both patients. Although a gingival biopsy in case 1 did not show ${ }^{\infty}$ the hyaline thrombi of TTP, granular staining for $\vec{\circ}$ both IgM and IgG characteristic of SLE was noted $\overrightarrow{\vec{\omega}}$ along the basement membrane by direct immunofluorescence. Similar deposits of $\mathrm{IgM}$ and $\mathrm{IgG}$ were seen in biopsy specimens of both sun exposed and non-sun exposed skin. The splenectomy specimen or showed both the periarterial fibrosis of SLE and $\dot{\omega}$ scattered hyaline thrombi typical of TTP (Fig. 1). In $\frac{\omega}{6}$

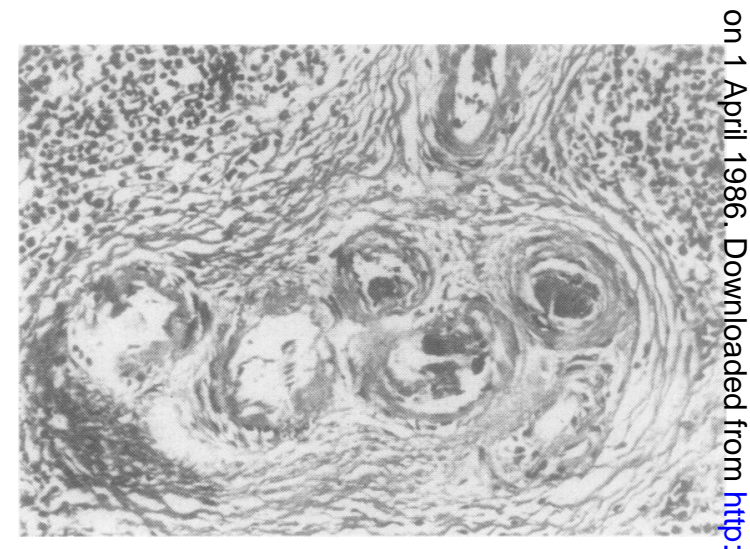

Fig. 1 Spleen of case 1. Arterioles within the white pulp display features of both TTP (intraluminal and subendothelial hyaline thrombi) and SLE (periadventitial fibrosis). (Haematoxylin and eosin stain, $\times 80$ ).

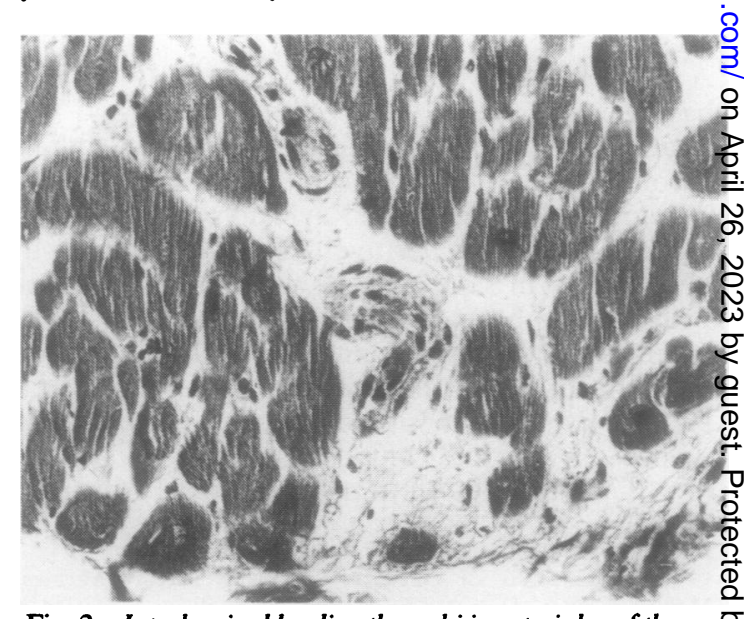

Fig. 2 Intraluminal hyaline thrombi in arterioles of the heart in case 2. (Haematoxylin and eosin stain, $\times 80$ ). 
case 2 the gingival biopsy specimen contained both intraluminal and subendothelial thrombi in a subepithelial arteriole. There was no significant stromal inflammation. At autopsy, in addition to membranous glomerulonephritis and splenic periarterial fibrosis characteristic of SLE, there were intraluminal and subendothelial hyaline thrombi within small arteries and arterioles of many organs, including heart, liver, spleen, kidneys, adrenals, lymph nodes, thyroid, pancreas, intestines, appendix, pituitary, choroid plexus, and brain. There was no evidence of vasculitis. The thrombi were especially prominent in the heart (Fig. 2). Although no definite myocardial infarction was identified grossly or microscopically, focal areas of red blood cell extravasation were seen.

\section{Discussion}

In this report we describe two patients with SLE who also developed TTP. Both patients fulfilled the 1982 American Rheumatism Association (ARA) criteria for the classification of $\mathrm{SLE}^{4}$ and showed splenic periadventitial fibrosis. Both developed syndromes characteristic of TTP and were found to have multiple visceral subendothelial hyaline thrombi. Gingival biopsies, when positive, are helpful in confirming the diagnosis of TTP, but they lack both sensitivity and specificity. ${ }^{56}$ Similarly, the finding of hyaline thrombi in small arteries and arterioles in the spleen and in other organs, though characteristic of TTP, may be present in other disorders, especially disseminated intravascular coagulation (DIC). Various histological features have been proposed as relatively specific markers for TTP. These include subendothelial (as opposed to only intraluminal) thrombi, microaneurysm formation, and severe endothelial cell proliferation within microaneurysms (creating so called 'glomeruloid bodies'). These have not been shown to be reliable in differentiating TTP from DIC pathologically. ${ }^{78}$ Concentric adventitial fibrosis around central and penicilliary arteries of the spleen, especially when diffuse and severe, can be considered pathognomonic of SLE. ${ }^{9-11}$ Coexistence of these 'onion skinning' lesions with hyaline thrombi in the spleen has been reported in cases of SLE associated with TTP. ${ }^{12-14}$

When prior reports of the association of SLE and TTP ${ }^{12}{ }^{13}{ }^{15-19}$ are analysed by the 1982 ARA criteria for SLE only four patients can be identified who had unequivocal SLE clinically and subsequently developed TTP. ${ }^{12} 131819$ A fifth patient developed both SLE and TTP concurrently. ${ }^{12}$ In addition to the small number of cases in which the coexistence of SLE and TTP can be considered well established, there are additional reports linking SLE and TTP in which either the clinical or the pathological evidence for SLE is not conclusive. ${ }^{14-17}$ Levine and Shearn reviewed 151 cases of TTP reported before 1964 and noted that 34 patients $(23 \%)$ had pathological finding suggestive of SLE. ${ }^{14}$ In most of these cases, however, Libman-Sacks endocarditis was the sole finding thought to indicate SLE. Nevertheless, eight patients did have splenic periadventitial fibrosis, seven had evidence of proliferative glomerulonephritis, and five had a positive LE cell test. Clinical information is incomplete for many of the patients, but it is likely that some did indeed have both SLE and TTP.

Recently Dixit et al reported a patient with probable SLE, C2 deficiency, and relapsing TTP, though the diagnosis of TTP was not confirmed histologically. ${ }^{20}$ One of our two patients presented with hypocomplementaemia at the time of her initial episode of TTP but had a normal total haemolytic complement, $\mathrm{C} 2, \mathrm{C} 3$, and $\mathrm{C} 4$ on other occasions. Our other patient had normal complement levels at the time of her TTP despite previous periods of hypocomplementaemia in conjunction with activity of her SLE. Thus it appears that in patients with SLE and TTP the complement profile is quite heterogeneous.

Although TTP occasionally complicates the course of SLE, the pathogenesis of TTP in these patients is not well understood. Patients with SLE may develop disturbances in blood coagulation, ${ }^{21}$ and lupus patients with circulating anticoagulants may be at risk for thrombotic events. ${ }^{22}$ Some patients with SLE who developed TTP had a history of prior thrombophlebitis. ${ }^{12}{ }^{19}$ As yet, the relationship of these observations to the occurrence of TTP in SLE is unclear. In our patients a lupus anticoagulant was not detected and the titre of anticardiolipin antibodies was not determined.

There is little evidence connecting the immunological abnormalities of SLE with the occurrence of TTP. Circulating immune complexes had been detected during a prior flare of SLE in one report but were absent when TTP developed. ${ }^{13}$ In that patient SLE was clinically inactive when TTP occurred, but other patients have developed TTP during a flare of SLE. ${ }^{12} 19$

The diagnosis of TTP may be difficult and particular problems arise in patients with SLE because of the overlapping manifestations of the two disorders. Anaemia, thrombocytopenia, fever, neurological abnormalities, and renal disease all occur in SLE as well as in TTP, but a microangiopathic peripheral blood smear should alert the clinician to the possibility of TTP.

The prognosis of patients with TTP is guarded, 
even when corticosteroids and splenectomy are employed. ${ }^{12}$ The majority of patients previously reported with TTP and SLE died of TTP. The introduction of plasmapheresis or plasma infusion (or both) and the use of antiplatelet agents appears to have improved the management of TTP, but relapses may occur despite good initial responses. Our first patient had a chronic course, required splenectomy, and eventually stabilised on cyclophosphamide. The second patient relapsed and died after discontinuing her medications. Microthrombi in the heart, similar to those seen in case 2, may account for cardiac death in TTP in the absence of either clinical or pathological signs of myocardial ischaemia, perhaps by affecting the conducting system. $^{23}$

Dr Fox was supported by a postdoctoral fellowship of the Arthritis Foundation.

\section{References}

1 Amorosi E I, Ultmann J E. Thrombotic thrombocytopenic purpura: report of 16 cases and review of the literature. Medicine (Baltimore) 1966; 45: 139-53.

2 Ridolfi R L, Bell W R. Thrombotic thrombocytopenic purpura: report of 25 cases and review of the literature. Medicine (Baltimore) 1981: 60: 413-28.

3 Cuttner J. Thrombotic thrombocytopenic purpura: a ten-year experience. Blood 1980; 56: 302-6.

4 Tan E M, Cohen A S, Fries J F, et al. The 1982 revised criteria for the classification of systemic lupus erythematosus. Arthritis Rheum 1982; 25: 1271-7.

5 Goldfard P B. Finch S C. Thrombotic thrombocytopenic purpura. A ten year study. JAMA 1978: 226: 644-7.

6 Goodman A. Ramas R. Petrelli M. Hirsch S A. Bukowski R, Harris J S. Gingival biopsy in thrombotic thrombocytopenic purpura. Ann Intern Med 1978; 89: 501-4.

7 Umlas J, Kaiser J. Thrombohemolytic thrombocytopenic purpura (TTP). A disease or a syndrome? Am J Med 1970; 49: 723-8.

8 Cryer P E, Kissane J M, eds. Clinicopathologic Conference. Am J Med 1977; 63: 789-98.
9 Klemperer P. Pollack A D. Baehr G. Pathology of disseminated lupus erythematosus. Arch Pathol 1941: 32: 569-631. ?

$10 \mathrm{Kaiser} I \mathrm{H}$. Specificity of periarterial fibrosis of the spleen in disseminated lupus ervthematosus. Bull Johns Hopk Hosp 1942: 71: 31-43.

11 Eversole S L. Cases of disseminated lupus erythematosus diagnosed as idiopathic thrombocytopenic purpura. Bull Johns Hopk Hosp 1955: 96: 210-22.

12 Dekker A. O'Brien M E. Cammarata R J. The association of thrombotic thrombocytopenic purpura with systemic lupuson erythematosus: a report of two cases with successful treatment ${ }^{\text {s }}$ of one. Am J Med Sci 1974: 267: 243-9.

13 Cecere F A. Yoshinoya S. Pope R M. Fatal thromboticthrombocytopenic purpura in a patient with systemic lupus $\vec{\omega}$ erythematosus. Relationship to circulating immune complexes. Arthritis Rheum 1981: 24: 550-3.

14 Levine S. Shearn M A. Thrombotic thrombocytopenic purpura and systemic lupus erythematosus. Arch Intern Med 1964: 113: 826-36.

15 Laszlo M H. Alvarez A. Feldman F. The association of thrombotic thrombocytopenic purpura and disseminated lupus $\overrightarrow{0}$ erythematosus: report of a case. Ann Intern Med 1955; 42: $1308-20$.

16 Siegel B M. Friedman I A, Kessler S. Schwartz S O.Thrombotic thrombocytopenic purpura and lupus erythemato- $D$ sus. Ann Intern Med 1957: 47: 1022-9.

17 Ramkissoon R A. Thrombotic thrombocytopenic purpura and systemic lupus erythematosus. Calif Med 1966; 104: 212-4. $\overrightarrow{0}$

18 Alpert L I. Thrombotic thrombocytopenic purpura and sys- $\infty$ temic lupus erythematosus: report of a case with immunofluorescence investigation of vascular lesions. J Mount Sinai Hosp NY 1968; 35: 165-73.

19 Oen K. Petty R E, Schroeder M L, Briggs E J N, Bishop A J. Thrombotic thrombocytopenic purpura in a girl with systemic lupus erythematosus. J Rheumatol 1980; 7: 727-9.

20 Dixit R, Krieg A M. Atkinson J P. Thrombotic thrombocy topenic purpura developing during pregnancy in a C2-deficien patient with a history of systemic lupus erythematosus. Arthritio Rheum 1985: 28: 341-4.

21 Lee S L. Miotti A B. Disorders of hemostatic function in patients with systemic lupus erythematosus. Semin Arthritiso Rheum 1975; 4: 241-52.

22 Meuh J R. Herbst K D. Rapaport S I. Thrombosis in patients with the lupus anticoagulant. Ann Intern Med 1980: 92: 156-9.

23 Ridolfi R L, Hutchins G M. Bell W R. The heart and cardiac conduction system in thrombotic thrombocytopenic purpura. clinicopathologic study of 17 autopsied patients. Ann Interno med 1979; 91: 357-63. 